\title{
Subsequent risk of cancer among women with a history of placental abruption
}

\author{
Outi Riihimäki ${ }^{1}$, Minna Tikkanen ${ }^{1}$, Johanna Melin², Jorma Paavonen ${ }^{1}$, Mika Nuutila ${ }^{1}$ Mika Gissler ${ }^{3}$ \\ and Eero Pukkala² \\ ${ }^{1}$ Department of Obstetrics and Gynecology, University of Helsinki and Helsinki University \\ Hospital, Helsinki \\ 2 Finnish Cancer Registry, Institute for Statistical and Epidemiological Cancer Research, Helsinki, \\ Finland
3 THL National Institute for Health and Welfare, Helsinki

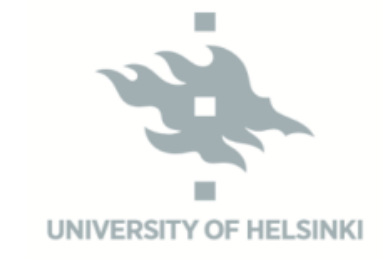

Introduction

Placentation is characterized by extensive cell proliferation and neovascularization. Similar phenomena occur in the development of cancer.

There is a growing body of evidence linking placental syndromes with long-term maternal morbidity and mortality. However, the link between placental abruption and subsequent cancer has not been well studied. Multiple risk factors or risk markers have been identified for placental abruption. Of these, smoking and alcohol use also expose to cancer.

Our aim was to describe the subsequent cancer risk among women with a history of placental abruption. Based on previous studies, we paid special interest on cancers of the lung and breast.

\section{Materials and Methods}

This was a historical prospective register-based cohort study. Data on women with placental abruption diagnosed between 1971 and $2005(n=7,804)$ were collected from the Finnish Hospital Discharge Register and the Finnish Medical Birth Register. The cohort was then linked with the Finnish Cancer Registry records dating up to the end of 2013. Standardized incidence ratios (SIRs) were calculated for different cancers by dividing the observed numbers of cancers by expected ones. The expected numbers were based on national cancer incidence rates.

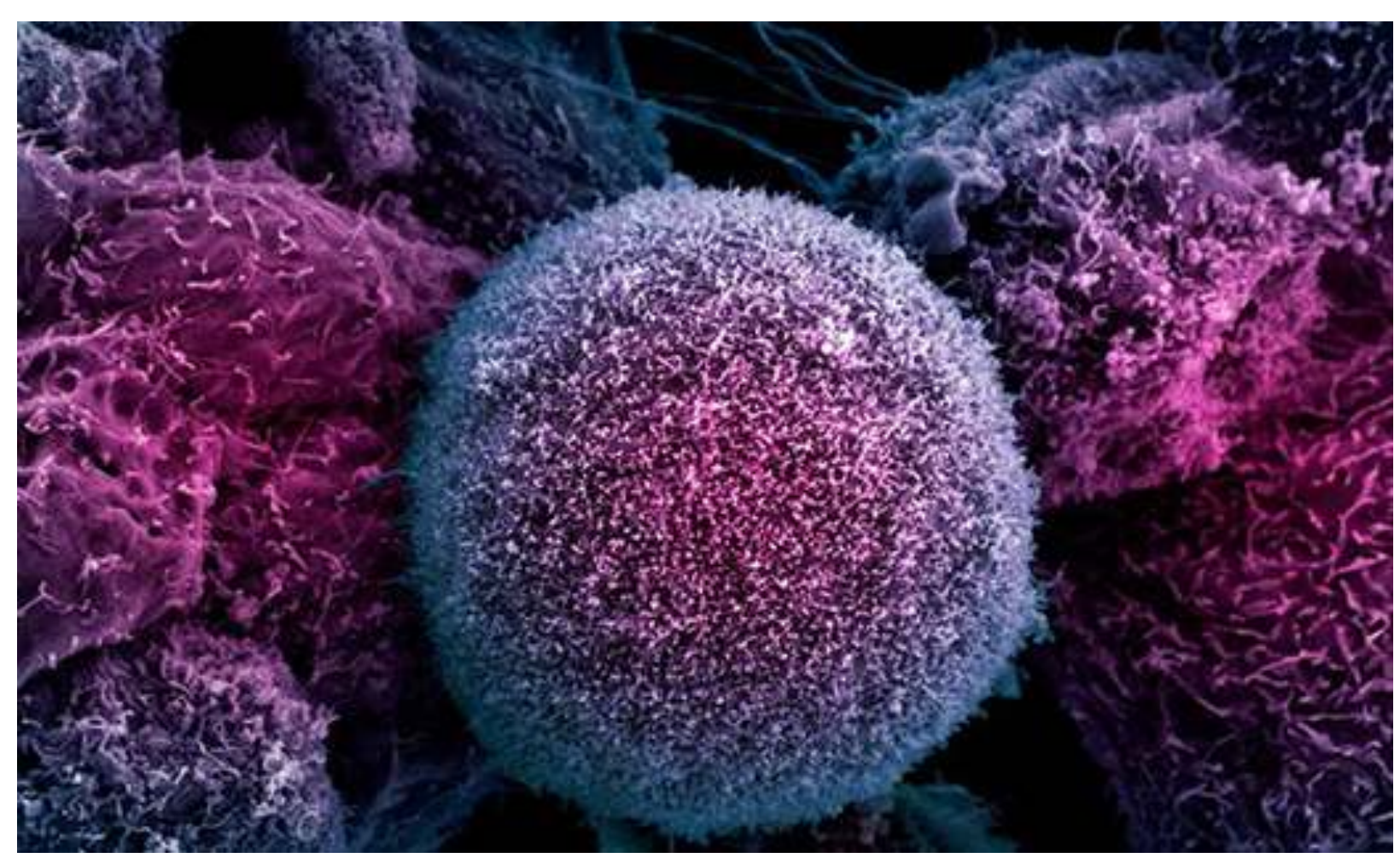

\section{Results}

During the follow-up, 597 cancers were found among women with a history of placental abruption. The overall risk of cancer was not increased (SIR 0.95, 95\% Cl 0.88-1.02). However, the history of placental abruption was associated with an increased risk of lung cancer (SIR 1.51, 95\% CI 1.05-2.10) and thyroid cancer (SIR $1.47,95 \% \mathrm{Cl} 1.04-2.02)$. A decreased risk was found for breast cancer (SIR 0.85, 95\% CI 0.75-0.96). The risk of rectal cancer was also decreased, although the numbers were small (SIR 0.49, 95\% Cl 0.20-1.01).

Table 1. Observed (Obs) and expected (Exp) numbers of cancer cases and standardized incidence ratios| (SIR) with 95\% confidence intervals (Cl) among women with a history of placental abruption during 19712013.

\begin{tabular}{|c|c|c|c|c|}
\hline Cancer site & Obs & Exp & SIR & $95 \% \mathrm{Cl}$ \\
\hline Any site & 597 & 628 & 0.95 & $0.88-1.02$ \\
\hline Stomach & 8 & 10.87 & 0.74 & $0.32-1.44$ \\
\hline Colon & 29 & 24.76 & 1.17 & $0.78-1.68$ \\
\hline Rectum & 7 & 14.18 & 0.49 & $0.20-1.01$ \\
\hline Pancreas & 9 & 11.83 & 0.76 & $0.35-1.44$ \\
\hline Lung, trachea & 35 & 23.15 & 1.51 & $1.05-2.10$ \\
\hline Melanoma of the skin & 18 & 27.08 & 0.66 & $0.39-1.05$ \\
\hline Skin, non-melanoma & 12 & 8.20 & 1.46 & $0.76-2.55$ \\
\hline Soft tissues & 6 & 4.65 & 1.29 & $0.47-2.80$ \\
\hline Breast & 234 & 273.85 & 0.85 & $0.75-0.96$ \\
\hline Cervix uteri & 17 & 12.38 & 1.37 & $0.80-2.19$ \\
\hline Corpus uteri & 25 & 31.80 & 0.79 & $0.51-1.16$ \\
\hline Ovary & 18 & 25.43 & 0.71 & $0.42-1.11$ \\
\hline Other female genital & 7 & 5.73 & 1.22 & $0.49-2.51$ \\
\hline Kidney & 15 & 11.98 & 1.25 & $0.70-2.06$ \\
\hline Bladder, urinary tract & 6 & 5.94 & 1.01 & $0.37-2.19$ \\
\hline Brain, central nervous system & 36 & 35.45 & 1.02 & $0.71-1.40$ \\
\hline Thyroid gland & 37 & 25.14 & 1.47 & $1.04-2.02$ \\
\hline Leukaemia & 15 & 9.14 & 1.64 & $0.92-2.70$ \\
\hline Hodgkin lymphoma & 7 & 3.52 & 1.99 & $0.80-4.09$ \\
\hline Non-Hodgkin lymphoma & 17 & 20.91 & 0.81 & $0.47-1.30$ \\
\hline
\end{tabular}

Conclusion: In women with a history of placental abruption the risk of lung cancer and thyroid cancer were increased and the risk of breast cancer was decreased. These observations can be explained to some extent by risk factors or risk markers for placental abruption. 